\title{
ROLE OF MICRO ESR IN THE EVALUATION OF NEONATAL SEPSIS
}

Raju Kafle, Jaydeep Yadav, ${ }^{1}$ Binod Kumar Gupta ${ }^{1}$, Badri Kumar Gupta ${ }^{1}$

\begin{abstract}
\section{INTRODUCTION}

In a developing country like Nepal, where proper medical care and obstetrical facilities are still scarce in the rural areas, neonatal sepsis is a major cause of neonatal morbidity and mortality. With limited resources, early diagnosis and treatment if crucial, may not be achieved. Objective of this study is to evaluate the role of micro ESR in the diagnosis of neonatal sepsis.
\end{abstract}

\section{MATERIAL AND METHODS}

A prospective analytical study was done where all neonates with suspected sepsis admitted in NICU were included in the study. Micro-ESR was measured with relevant septic screening investigations. The micro-ESR was compared with age specific cut off value and the results were compared with various clinical presentations, laboratory findings and outcome variables.

\section{RESULTS}

Out of 250 neonates, micro-ESR was elevated in $12 \%$ (29 neonates) of cases. Total proven sepsis is $6 \%$, probable sepsis $60.8 \%$ and no sepsis in $33.2 \%$. Correlation of elevated micro ESR was statistically significant with presence of clinical symptoms, clinical and systemic signs. It had significant clinical association with blood culture and CSF findings. The sensitivity, specificity and positive and negative predictive value of micro ESR compared to blood culture was $93.3 \%$, $93.6 \%, 48.3 \%$ and $99.5 \%$.

\section{CONCLUSION}

Micro ESR is a simple, relatively cheap and sensitive study in prediction of neonatal sepsis. It can be useful test in settings with limited resources.

\section{KEYWORDS Micro ESR, Neonate, Sepsis}

1. Department of Paediatrics, Universal College of Medical Sciences, Bhairahawa, Nepal.

DOI: http//doi.org/10.3126/jucms.v7i2.27131

For Correspondence

Dr. Raju Kafle

Department of Paediatrics

Universal College of Medical Sciences

Bhairahawa, Nepal

Email:drrajukafle2@gmail.com 


\section{INTRODUCTION}

Sepsis is the commonest cause of neonatal mortality; it is responsible for about $30-50 \%$ of the total neonatal deaths in developing countries. ${ }^{1}$ It is estimated that up to $20 \%$ of neonates develop sepsis and approximately $1 \%$ die of sepsis related causes. ${ }^{2}$ All neonates suspected to have sepsis should have a septic screen to corroborate the diagnosis. However, the decision to start antibiotics need not be conditional to the sepsis screen result, if there is a strong clinical suspicion of sepsis.

The clinical diagnosis of neonatal sepsis is difficult because the signs and symptoms are not always specific. There is no laboratory test with $100 \%$ sensitivity and specificity. ${ }^{3}$ Blood culture has been considered the gold standard for confirmation of diagnosis but the results are available only after 48-72 hours. Moreover, in many cases blood culture fails to detect the offending organism/bacteria. So, the search for a reliable test continues, especially one that is useful in culture-negative cases. The erythrocyte sedimentation rate (ESR) is a useful test applied to screening for, or the serial monitoring of disease states. ${ }^{4}$ Determination of ESR in capillary blood can be of value in evaluating sick newborns. ${ }^{5}$ The micro method is simple to perform and reproducible and requires only $0.2 \mathrm{ml}$ blood. $^{4}$

Though being simple and oldest bed side test Micro ESR is not routinely measured and very less study has been done in Nepalese Population. This is an attempt to see the predictive value of this test. If it shows good predictive value physicians working at settings with limited resources can benefit and contribute in early diagnosis and prompt treatment of sepsis thus reducing neonatal morbidity and mortality. Hence, we planned this study to identify cost-effective test like Micro ESR for the diagnosis of neonatal sepsis so that prompt treatment can be initiated and neonatal deaths can be minimized.

\section{MATERIAL AND METHODS}

This was a prospective analytical study carried out at Neonate unit and Maternity ward of Universal college of Medical Sciences, Bhairahawa, Rupandehi Nepal. With the absolute error/precision of 5\% (corresponding to a confidence interval of $50 \% \pm 5 \%$ ) at type 1 error of $5 \%$ the sample size was calculated from the prevalence of $19 \%$ from the previous studies $^{6}$ and this size comes out to be 236 but we have taken a total of 250 babies admitted with diagnosis of suspected sepsis fulfilling the inclusion and exclusion criteria were included in the study from April 2016 till April 2017. Inclusion criteria was neonates admitted as suspected neonatal sepsis and with $\mathrm{Hb}>10 \mathrm{Gm} \%$ and $<20 \mathrm{Gm} \%$. Exclusion criteria were anemia $(\mathrm{Hb}<10 \mathrm{Gm} \%)$ and polycythemia $(\mathrm{Hb}>20 \mathrm{Gm} \%)$, presence of a chromosomal, genetic, or inborn metabolic disorder, neonates already on treatment and neonates without risk factors and clinical criteria for sepsis. Ethical approval was taken from Institutional Review Board before conducting the study.

Neonates suspected of sepsis were enrolled in the study after getting written consent from the care givers or parents. During the septic work up of the baby minimum amount of blood $(0.2$ $\mathrm{ml}$ ) is collected in a capillary tube using full aseptic precautions and micro ESR is read at one hour. The treatment of the baby was continued as per the protocol irrespective of micro ESR values.

The neonates were evaluated by a thorough history from mother, maternal parameters at birth and detail clinical examinations. The weight of the babies was taken using electronic scale with calibration of 50 grams. Gestational assessment was done using Modified Ballard Score.

The risk factors for sepsis were premature rupture of membranes, foul smelling liquor, maternal fever during 24 hours of delivery, maternal chorioamnionitis, foul smelling liquor and meconium staining of liquor. The neonatal risk factors included babies with refusal to feed, fever, lethargy, tachypnea, hypothermia, breathing difficulty, eye discharge, jaundice, skin pustules and any other signs of sepsis.

The laboratory and radiological investigations were done for diagnosis and confirmation of the diagnosis and confirmation of infection as per clinical scenario. Complete blood count including peripheral smear for band cells, toxic granulations and degenerative changes in neutrophils, blood culture was sent and relevant radiological investigation and CSF examination was done. Relevant swab and surface and discharge cultures like skin pustule, eye swab, culture of stool and urine when indicated and tracheal aspirate in cases of ventilated babies. Stool culture was sent in babies with persistent abdominal distension and significant nasogastric aspirate or persistent vomiting. Neonates were classified as proven sepsis for those with culture proven sepsis, probable sepsis for those with clinical impression of sepsis with other abnormal laboratory or radiological results but negative for culture and no sepsis when clinical parameters, lab or radiological parameters all were normal.

Antibiotics were started as per the Unit protocol on the babies of clinical presentation or obstetric risk factors. Initial empiric antibiotics used were cefotaxime and gentamycin. Antibiotics were changed according to sensitivity pattern in cases of culture positive sepsis and on clinical grounds in cases of culture negative sepsis. Babies were followed till discharge.

Measurement of Micro ESR was performed using $0.2 \mathrm{ml}$ 
blood in microhematocrit capillary tubes in all babies suspected of sepsis and the readings are read at one hour of the test and labeled as normal to age or elevated for age based on the conventional values age in day of life +3 being the $95^{\text {th }}$ percentile value as given by Alder and Denton in 1975 . $^{7}$ The babies continued to get treatment as per the protocol irrespective of the micro ESR Values.

The collected data was entered into SPSS software and analysis was also done by the same. Main statistical methods used were cross tabulation and comparison using micro ESR as a dependent variable and other variables as independent variable. Pearson's Chi square test was applied to test the statistical significance, value of $<0.05$ is considered significant in statistical analysis.

\section{RESULTS}

Out of 250 babies, micro ESR was elevated in $29(12 \%)$ babies and blood culture was positive in $15(6 \%)$ cases and meningitis was present in two cases but CSF culture was positive in none of the cases. Total culture proven cases were $15(6 \%)$ only as shown in Figure 1. Probable sepsis based on other laboratory abnormalities and clinical features (Figure 2) were $152(60.80 \%)$.

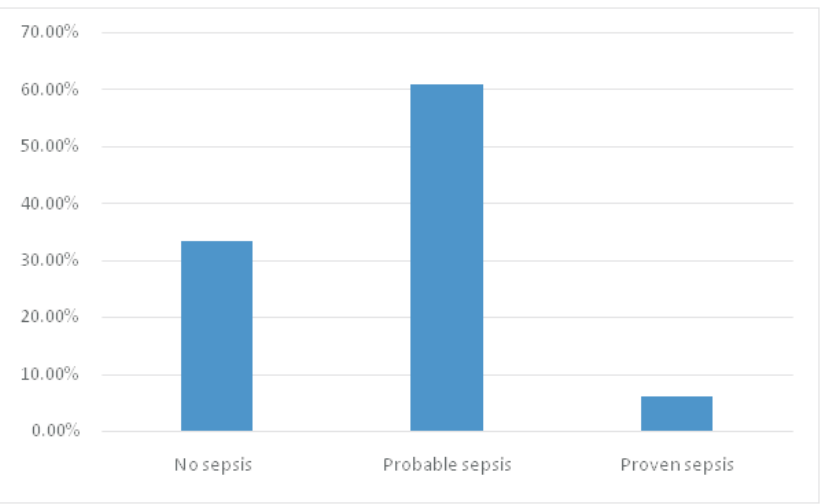

Figure 1. Showing percentage of various sepsis

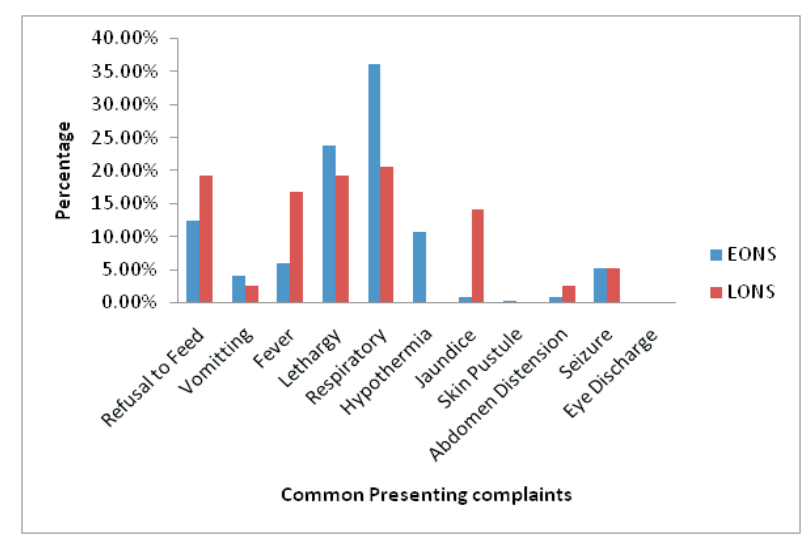

Figure 2. Common presenting complaints of babies
Sixty seven neonates were included in the study because of obstetric risk factors without any clinical symptoms, however 183 neonates had clinical symptoms of sepsis. Of the clinical symptoms fast breathing, lethargy and refusal to feed were commonest three clinical symptoms at presentation as shown in Figure 2.

Correlation of elevated micro ESR was statistically significant with period of gestation, age at admission, presence of clinical symptoms, clinical signs and systemic signs. The elevated micro ESR showed significant clinical association with clinical sepsis scoring, hematological scoring system and blood culture and CSF findings at $\mathrm{P}$ value of $<0.05$.

The blood culture was positive only in 15 babies ( $6 \%$ of cases) in our study and. E coli was grown in six cases, S.aureus in four cases, Pseudomonas in three cases, and coagulase negative staphylococci (CONS) and Klebsiella pneumoniae in one case each as shown in Table 1.

\section{Table 1. Blood culture report in our study}

\begin{tabular}{lcc}
\hline Bacteria & Count & Percentage \\
\hline E. coli & 6 & $2.4 \%$ \\
S. aureus & 4 & $1.67 \%$ \\
Pseudomonas & 3 & $1.2 \%$ \\
Klebsiella & 1 & $4 \%$ \\
CONS & 1 & $4 \%$ \\
No growth & 235 & $94.0 \%$ \\
\hline
\end{tabular}

The sensitivity, specificity and positive and negative predictive value of micro ESR compared with blood culture was $93.3 \%, 93.6 \%, 48.3 \%$ and $99.5 \%$ respectively.

The performance of micro ESR in diagnosing probable sepsis in comparison to proven or no sepsis cases was with sensitivity, specificity and positive and negative predictive value of $33.3 \%, 98.8 \%, 66.7 \%$ and $1.2 \%$ respectively.

\section{DISCUSSION}

The World Health Organization estimates that perinatal deaths are responsible for most of the childhood mortality below the age of five years in the developing countries. ${ }^{8}$ Neonatal infections are by far the most common cause of such perinatal mortality. ${ }^{9}$ Considering the high mortality and serious morbidity among neonates suffering from sepsis, a diagnostic marker with high sensitivity and specificity is desirable. However, there is no laboratory marker that has all the characteristics of an ideal infection marker. Interleukin-6 (IL6) and interleukin-8, CD11b, and procalcitonin (PCT) are all sensitive markers of infections. PCT is also a very useful 
marker for diagnosis of early-onset neonatal sepsis (EONS). In very-low-birth-weight neonates, a serum PCT value $>2.4$ $\mathrm{ng} / \mathrm{ml}$ justifies early empirical antibiotic therapy, while in normal-birth-weight infants a PCT value of $0.5-2.4 \mathrm{ng} / \mathrm{ml}$ indicates neonatal sepsis. ${ }^{10}$ However, these sensitive markers of infections are not available all the times in most of our center within the country and also these sensitive tests are not equally cost effective. With the increasing facilities and the use of other septic screening tools as above mentioned micro ESR being a single, cheap and less time consuming should be reassessed and redefined in our setting.

The common predisposing obstetric risks for sepsis in our study were PROM $>18$ hours which comprised of $14.5 \%$ in early onset sepsis and 50\% in late onset sepsis and another second common risk factor as meconium stained liquor in $63.2 \%$ and $50 \%$ respectively in early and late onset sepsis. This observation is higher than that studied in various other places. In a similar study done by Joshi BD $25.9 \%$ of cases of sepsis were associated with PROM of $>24$ hours. ${ }^{11}$ The common neonatal risk factor for sepsis in our study is respiratory problem, lethargy and refusal to feed which is similar to other studies. ${ }^{11}$

Blood culture, the gold standard test for diagnosis of neonatal sepsis was positive in $15(6.0 \%)$ babies in the study and CSF culture was positive in only three $(1.2 \%)$ babies. Other cultures like culture of tracheal secretions in ventilated babies was positive in one, stool culture was positive in two and eye swab was positive in only one of the babies. Thus, the total culture positive were 15 cases only. The blood culture positivity was $10.2 \%$ and CSF positivity was $5.45 \%$. In a study done by Kaiser JR et al the blood culture positivity correlates well with this study but the low turnout of CSF culture could be due to delayed lumbar puncture or delayed processing of CSF in sick babies in our study. ${ }^{12}$

The present study showed that micro ESR correctly identified 14 out of 15 neonates who had blood culture proven sepsis giving a sensitivity of $93.3 \%$. The sensitivity obtained in this study was lower than the $96.9 \%$ documented by Okolo et al in Benin $^{13}$ who used micro ESR values above the $95^{\text {th }}$ percentiles. Micro ESR was unable to identify one out of 15 neonates who had blood culture proven sepsis giving a specificity of $93.6 \%$. This is at variance with $60.9 \%, 75 \%$ and $90 \%$ reported by Diwakar and Rosul et al ${ }^{14}$ and Okolo et al. ${ }^{13}$ The variation in the specificity in this study as compared to latter studies could be also attributed to the different diagnostic criteria and the different cut off values used compared to present study.

The positive predictive value of micro ESR compared to blood culture is $48.3 \%$ as obtained in the present study. This value is much higher than the $13.8 \%$ reported by Zeeshan et al. ${ }^{6}$ The difference could be attributed to the disparity in the cut off values used. The negative predictive value of $99.5 \%$ is obtained in the present study. This value is near to value reported by Okolo et al. ${ }^{13}$ This value is higher than reported by Zeeshan et al. ${ }^{6}$ In this latter study done by Zeeshan et al children aged 1-3 months constitute the study population as opposed to present study where neonates aged 0-28 days were included.

Blood culture positivity was $25 \%$ in studies done by Mathur et $\mathrm{al}^{15}$ and Joshi et al. ${ }^{11}$ Highest blood culture positive rates of up to $59.7 \%$ was observed in a study done by Karki et al. ${ }^{16}$ The lower culture positive results $(6 \%)$ in our study could be due to the fact that most of the included neonates were $<24$ hours of age and blood culture was sent at the time of first examination as screening procedure before they developed any signs of sepsis clinically. Of the 15 blood culture positive cases in our study Gram negative bacilli Escherichia coli was isolated in six $(2.4 \%)$ cases followed by Staphylococcus aureus in four (1.67\%), Pseudomonas in three $(1.2 \%)$, Klebsiella pneumoniae in one $(0.4 \%)$ case, CONS in one case $(0.4 \%)$. This is similar to a study done by Ghosh et $\mathrm{al}^{17}$ where the commonest organism isolated was E.coli $9(30 \%)$ followed by Staphylococcus aureus 8 (27\%) and Klebsiella 5 (17\%). While Kumar and Singhi ${ }^{18}$ isolated Klebsiella as the commonest organism followed by Staphylococcus aureus, Pseudomonas and E. coli.

\section{CONCLUSION}

Micro ESR is a simple cheap and relatively sensitive, specific with good negative predictive value in the prediction of neonatal sepsis and can be useful test in the settings with limited resources.

\section{Permission from IRB: Yes}

Conflicting interests: None

Funding: None

\section{REFERENCES}

1. Bang AT, Bang RA, Bactule SB, Reddy HM, Deshmukh MD. Effect of home-based neonatal care and management of sepsis on neonatal mortality: field trial in rural India. Lancet.1999; 354:1955-61.

2. Stoll BJ. The global impact of neonatal infection. Clin Perinatol 1997; 24:1-21.

3. Plazek MM, White LA. Early and late neonatal septicemia. Arch Dis Child. 1983;58:728-31.

4. Barret BA and Hill. A micro method for the Erythrocyte 
Sedimentation Rate suitable for use on venous or capillary blood. J Clin Pathol.1980;33:1118-20.

5. Shah Yogesh and Kumar Ashir. The Erythrocyte Sedimentation Rate: Evaluation of Micro Technique. Journal of the National Medical Association: 1982:74(9): 887-9.

6. Zeeshan A, Tariq G, Talal W, Salman A, Shahid A, Shahid M. Diagnostic value of C-reactive protein and other haematological parameters in neonatal sepsis. J Coll Physicians Surg Pak. 2005;15:152-6.

7. Adler SM, Denton RL. The Erytrhrocyte sedimentation Rate in newborn period. Journal of Pediatrics. 1975; 8(6):942-8.

8. Geneva: World Health Organization; 2005. WHO. World Health Report.

9. Largo MG, Stekelenburg J. The millennium project of the United Nations, focusing on adequate postpartum care to reduce maternal and neonatal mortality world-wide. Ned Tijdschr Geneeskd. 2006;150:11437.

10. Auriti C, Fiscarelli E, Ronchetti MP, Argentieri M, Marrocco G, Quondamcarlo A, Seganti G, Bagnoli F, Buonocore G, Serra G, Vacolla G, . Procalcitonin in detecting neonatal nosocomial sepsis. Archives of Disease in childhood- Fetal and Neonatal Editon. 2012 Sep 1;97 (S) F368-70

11. Joshi BD Clinico-laboratory Profile of Neonatal Septicemia. Thesis for the Degree of Doctor OF Medicine (Pediatric and Adolescent Medicine); B.P. Koirala Institute of Health Sciences, Dharan.2002.

12. Kaiser JR, Casat je, Lewno MJ. Sensitivity of 48-hour cultures (abstract). In: Kairalla AB (edtrs): Neonatal Journal Club. Journal of Perinatology. 2002;22(6): 445-7.

13. Okolo AA, Scott-Emuakpor AB, Omene JA. The diagnostic value of leukocyte indices and micro-erythrocyte sedimentation rate in neonatal infections. Trop Geogr Med. 1988;40:304-8

14. Diwakar KK, Rosul G) Revised look at micro erythrocyte sedimentation rate in neonates. Indian Pediatr.1999;36:703705 .

15. Mathur M, Shah H, Dixit K, Khambadkones, Chakrapani A. Bacteriological Profile of Neonatal Septicemia Cases for the Year 1990-1991. Journal of Postgraduate Medicine. 1994;40 (1):18-20.

16. Karki BM, Parija SC. Analysis of Blood culture Isolates from Hospitalized neonates in Nepal. Southeast Asian Journal of Tropical Medicine and Public Health. 1999;30(3):546-8.

17. Ghosh S, Mittal M, Jaganathan G. Early Diagnosis of Neonatal Sepsis using a Hematological Scoring System. Indian J Med Sci. 2001;55;495-550.

18. Kumar V, Singhi S. Predictors of serious bacterial infections in infants up to 8 weeks of age. Indian Pediatrics. 1994:31:171-80. 